\title{
Evaluation of Child Self-perception Regarding Their Oro-dental Status
}

\author{
Mártha I. Krisztina1, Roșu Sorana², Gyergyay Réka³, Vikárius Katalin4 \\ ${ }^{1}$ Department of Pediatric Dentistry and Orthodontics, Faculty of Dental Medicine, University of Medicine and Pharmacy, Tîrgu Mureș, Romania \\ 2 PhD student, "Grigore T. Popa" University of Medicine and Pharmacy, lași, Romania \\ 3 PhD student, University of Medicine and Pharmacy, Tîrgu Mureș, Romania \\ ${ }^{4}$ Student, University of Medicine and Pharmacy, Tîrgu Mureș, Romania
}

\begin{abstract}
Aim: The aim of this study was to evaluate the state of oral health and the self-perception of children regarding their oro-dental status, their knowledge and attitude towards oral health.

Methods: A sample of 130 children (11-14 years) and one of their parents were asked to complete a Child Perception Questionnaire (CPQ11-14) in Sfântu Gheorghe, Romania. From these, 69 children were examined and data was recorded about Decayed-Missing-Filled Teeth (DMFT), oral hygiene, dental malposition and malocclusion, Aesthetic Component of Index of Treatment Need (AC-IOTN). Consent of the parents was obtained. Statistical analyses were performed.

Results: The optimal score of CPQ evaluation is 96 and the maximum score achieved was 93, the minimum was 43.78 .10 average score can be graded as medium. As the data points out, most of the children and their parents have a medium knowledge and self-perception. The clinical examination underline the main problems: dental caries (62\%), occlusal coloration (23\%), dental malposition (17\%), rooftop deep bite (14\%), dental rotation and crowding (12\%), dental plaque and tartar (10\%). As far as the AC-IOTN is concerned, 48 of 69 children have no need for treatment, 18 have a high need for treatment, and 3 have a moderate need for treatment.

Conclusions: Overall, the children and their parents have a poor oral health knowledge, which is reflected in the medium level of the achieved $\mathrm{CPQ}$ scores, in the clinical examination and can be associated with high levels of dental caries and malocclusion.
\end{abstract}

Keywords: children, oral health, self-perception, CPQ11-14, IOTN

Received: 25 July 2013

\section{Introduction}

The present generation is much more preoccupied with their physical appearance than those before, because it is an important element in how the world perceives someone and it is a main factor in social integration. Every human being - adults and children - observe the world and themselves differently. They have different knowledge and opinion about what is beneficial, healthy or esthetic for them. From this point of view, every patient is unique and needs to be treated individually $[1,2]$. This can be realized only by using a wide range of techniques like questionnaires and indexes - CPQ11-14, IOTN, COHRQoL, DMFT- to enhance the outcome of diagnosis and treatment.

Preventive approach is one of the most important features of modern dentistry. Multiple medical, social and economic consequences of oral diseases and their treatment require the adoption of preventive measures to be addressed to a larger population, especially schoolchildren.

The purpose of our study was to evaluate the oral health status of children aged between 11-14 year old and their self-perception regarding their oro-dental condition. The main role was to establish if there are major problems that affect the oro-dental device and if they influence the general well-being or not.

Correspondence to: Krisztina Mártha

E-mail: marthakriszti@yahoo.com

\section{Material and methods}

In our study 130 children were surveyed, 67 boys and 63 girls, aged between 11-14 years. They are frequenting the $6^{\text {th }}, 7^{\text {th }}$ and $8^{\text {th }}$ grades in the "Székely Mikó" College in Sfântu Gheorghe, Romania.

Phase $1-130$ children and their parents were asked to fill in the CPQ11-14 [3], which contains 27 questions for children and 8 questions for their parents and their consent. The 35 questions are grouped in 4 categories - oral symptoms, functional limitation, emotional and social well-being. Each question has 5 possible answers from which one is chosen. Every response has a value from 0-4, 0 meaning low and 4 meaning excellent [3]. After grading each question, a score is calculated from the sum of all points. The score is compared to the ideal score of 96 .

Phase 2 - 69 children were examined and data was collected about oral hygiene, DMFT, malocclusion, dental malposition, AC of IOTN [4]. Here a group of 24 children presented different grades of malocclusion, dental malposition, rotation and crowding. Another group of 45 children didn't show any sign of malocclusion but they showed a wide range of dental caries, plaque and tartar. From the total of 69 patients examined only 10 did not present any affection. After completing all phases, some photographs were taken of the most severe cases. 
Table I. Interpretation of the CPQ 11-14 questionnarie and the comparison to the ideal value

\begin{tabular}{lc}
\hline Optimal score & $96(100 \%)$ \\
Maximal score & 93 \\
Minimal score & 46 \\
Average score & 78.10 \\
Urban average score & 78.92 \\
Rural average score & 74.22 \\
\hline
\end{tabular}

\section{Results}

The CPQ 11-14 questionnaire we used in this study seems to be a reliable and valid method for analysing the impact of oral and oro-facial conditions on 11- to 14-year-old children.

As Tables I and II show that the average score achieved is 78.10 , that compared to the optimal score of 96 can be graded as medium achievement. The evaluation could not highlight any significant difference between the average score by gender and age. A slightly bigger change can be seen between the scores of those who have no malocclusion (73.09) and those who don't (83.61). However, there is a discernible difference between male and female, where girls have an average score of 77.06 and the boys 79.08 .

Some differences in the frequency of dental lesions and malocclusion types can be found. The main problems are dental caries (62\%), marmoration in pits and fissures $(23 \%)$, dental malposition (17\%), rooftop deep bite $(14 \%)$, dental rotation and crowding (12\%) and finally dental plaque and tartar (10\%), as presented in Table III.

We found also certain differences in the frequency of anomalies regarding children's gender. Here we can underline the fact that girls have less problems than boys. Boys have a higher rate in dental caries (79\%), dental malposition $(24 \%)$, dental plaque and $\operatorname{tartar}(12 \%)$ than girls. Also there are more complex cases $(21 \%)$ than in the case of girls (14\%), see Table IV.

As far as the AC of IOTN is concerned, our results show that 48 of 69 children have no need for treatment,

Table III. The frequency of dental lesions, malocclusion

\begin{tabular}{lcc}
\hline Condition & $\begin{array}{c}\text { Total number } \\
\text { (of 69) }\end{array}$ & Percentage \\
\hline No problems & 5 & $7 \%$ \\
Dental plaque and tartar & 7 & $10 \%$ \\
Marmorations & 16 & $23 \%$ \\
Dental caries & 43 & $62 \%$ \\
Radicular remains & 5 & $7 \%$ \\
Extracted teeth & 2 & $3 \%$ \\
Anodontia & 2 & $3 \%$ \\
Diastemas & 3 & $4 \%$ \\
Dental rotation and crowding & 8 & $12 \%$ \\
Dental malposition & 12 & $17 \%$ \\
Edge bite & 2 & $3 \%$ \\
Rooftop deep bite & 10 & $14 \%$ \\
Covered deep bite & 4 & $6 \%$ \\
Reversed frontal bite & 1 & $1 \%$ \\
Open bite & 2 & $3 \%$ \\
\hline
\end{tabular}

Table II. Evaluation of the achieved scores in the CPQ11-14 questionnaire

\begin{tabular}{ll}
\hline Average scores by age & 11 year old -77.47 \\
& 12 year old -77.27 \\
& 13 year old -78.33 \\
& 14 year old -78.53 \\
Average scores by gender & Female -77.06 \\
& Male -79.08 \\
Average score for children with malocclusion & 73.09 \\
Average score for children with no problems & 83.61
\end{tabular}

18 have a high need for treatment and 3 have a moderate need for treatment. Analysis of the orthodontic treatment needs revealed a mean value of 2 of the IOTN, indicating reduced treatment need. Very high orthodontic treatment need was observed in $26 \%$ of the examined group, as it can be seen in Table V.

\section{Discussion}

Jokovic and al. demonstrated that in a sample consisting of 123 children of the mentioned age, mean CPQ11-14 scores were highest for oro-facial (31.4), lower for orthodontic (24.3), and lowest for pedodontic (23.3) patients. There were significant associations between the CPQ11-14 score and global ratings of oral health $(\mathrm{p}<0.05)$ and overall well-being $(\mathrm{p}<0.01)$ [5].

The interest in the psychological aspects of orthodontic treatment increases, but a drawback of many studies is that the psychological characteristics of the children themselves are often ignored. One of these psychological attributes is self-esteem (SE), which is a relatively stable personal resource that might moderate the effects of conditions or events. De Baets and al emphasised that in a group of 223 children (mean age 13.2 years) seeking for orthodontic treatment there was a significant relationship between orthodontic treatment need and oral health-related quality of life, and between self esteem and and oral health-related quality of life [6]. A more pronounced gap can be found between children with no affections (83.61)

Table IV. Differences in the frequency of anomalies regarding the children's gender

\begin{tabular}{lcc}
\hline Condition & Female & Male \\
\hline No problems & $14 \%$ & $0 \%$ \\
Dental plaque and tartar & $9 \%$ & $12 \%$ \\
Marmorations & $29 \%$ & $18 \%$ \\
Dental caries & $46 \%$ & $79 \%$ \\
Radicular remains & $3 \%$ & $12 \%$ \\
Extracted teeth & $3 \%$ & $3 \%$ \\
Anodontia & $6 \%$ & $0 \%$ \\
Diastemas & $6 \%$ & $3 \%$ \\
Dental rotation and crowding & $14 \%$ & $9 \%$ \\
Dental malposition & $11 \%$ & $24 \%$ \\
Edge bite & $6 \%$ & $0 \%$ \\
Rooftop deep bite & $14 \%$ & $15 \%$ \\
Covered deep bite & $9 \%$ & $3 \%$ \\
Reversed frontal bite & $3 \%$ & $0 \%$ \\
Open bite & $0 \%$ & $6 \%$ \\
Complex cases & $14 \%$ & $21 \%$ \\
\hline
\end{tabular}


Table V. The results of examination by the AC of IOTN

\begin{tabular}{llc}
\hline IOTN value & Meaning & $\begin{array}{c}\text { Total number } \\
\text { (of 69) }\end{array}$ \\
\hline IOTN 1-4 & No need for treatment & $48(70 \%)$ \\
IOTN 5-7 & Moderate need for treatment & $3(4 \%)$ \\
IOTN 8-10 & High need for treatment & $18(26 \%)$ \\
\hline
\end{tabular}

and those who have different degrees of malocclusion (73.09). The answers and the achieved scores show that kids have the capacity to auto evaluate themselves, only that this evaluation is subjective and is based on the low general knowledge they have about certain conditions. Overall, their lack of general knowledge about how oral health influences general health and life, do not realize the consequences that certain diseases, oral conditions have. If they don't have correct information, they can't make a totally correct self-evaluation.

Many psychological studies support the belief that selfesteem is a personal resource that facilitates coping with less favourable conditions such as poor dental aesthetics $[7,8]$. According to the Wilson-Clearly model, self-esteem is considered as a focal aspect of psychological health and higher levels of it would be related to greater life satisfaction $[9,10]$. Furthermore, several studies found a relationship between self-esteem and the way people are satisfied with their faces $[11,12,13]$.

Some limitations of the present study need to be considered. Our results demonstrated a correlation between orthodontic treatment need and oral health-related quality of life and between self-esteem and oral health-related quality of life in a clinical population. The question remains whether these correlations are still present in the general population.

The IOTN was used to determine the need for orthodontic treatment. One major disadvantage of this instrument is the risk of insensitivity for and misjudgement of the needs of the individual patient. It is hard to map the minor irregularities about which a patient is deeply concerned [14]. Another possible problem is that when orthodontic treatment need is based on the IOTN only, some patients who do not actually have psychosocial need for treatment would be treated $[15,16]$.

\section{Conclusions}

From the results of the survey we can conclude that the majority of children evaluated have low general knowledge about oral hygiene. This is due to the lack of general knowledge about the correct methods, additional elements of oral health and their role. Therefore, children suffer from dental plaque, tartar and caries. Also malocclusion has a negative impact on the quality of life of an adolescent.

The study also revealed that there is a great need for health education including prevention, hygiene and disease. The aim would be to draw the attention of children and parents on these increasing problems by permanent flow of information and evaluation. In lack of proper information or knowledge we can not expect a patient to ask for help.

During the examination we observed that these questionnaires and indexes are beneficial for the improvement of diagnostic and treatment methods. The AC of IOTN helps us to educate kids about different anomalies in order to improve their self-perception. It was observed that children can auto evaluate themselves better if they receive a photographic example to which they can compare their conditions. As a result, their evaluation is going to get better. The CPQ helps us to analyze the state of oral health and its functionality, the social and emotional state. As a result we can observe their influence on general health and life.

In order to prevent further increase of problems, we recommend the implementation of an extended preventive program that includes dentists, teachers, parents and children.

\section{References}

1. Harwood R, Miller SA, Vasta R. Psihologia copilului lași, Polinom, 2010, 326-329.

2. Mariana Păcurar, Doru Roman, Ilia Jipa. Ortodonție și estetică facială Târgu Mureș, University Press, 2012, 21-24.

3. Jokovic A, Locker D, Guyatt G. Short forms of the Child Perceptions Questionnaire for 11-14-year-old children (CPQ11-14): Development and initial evaluation. Health and Quality of Life Outcomes 2006;4:4.

4. Otuyemi OD, Kolawole KA. Perception of Orthodontic Treatment Need: Opinion Comparisons of Patients, Parents and Orthodontists. African Journal of Oral Health. 2005;2(1,2):42-51.

5. Corneagă A, Dănilă I, Balcoș C. Assessment of orthodontic treatment needs of schoolchildren from lasi according to Index of Treatment Needs (IOTN) and Dental Aesthetic Index (DAI). Romanian Journal of Oral Rehabilitation. 2011;3(4):23-27.

6. Jokovic A, Locker D, Stephens $M$, et al. Validity and Reliability of a Questionnaire for Measuring Child Oral-health-related Quality of Life. JDR. 2002;81(7):459-463.

7. De Baets E, Lambrechts H, Lemiere J, Diya L, Willems G. Impact of selfesteem on the relationship between orthodontic treatment need and oral health-related quality of life in 11- to 16-year-old children. Eur J Orthod. 2011. doi: 10.1093/ejo/cjr088

8. Harter S. Manual for the self-perception profile for adolescents. University of Denver, Denver, 1988, 7-11.

9. Agou S, Locker D, Streiner DL, Tompson B. Impact of self-esteem on the oral-health-related quality of life of children with malocclusion. American Journal of Orthodontics and Dentofacial Orthopedics. 2008;134:484-489.

10. Rosenberg M. Society and the adolescent self-image. Princeton University Press, Princeton, 1965, 305-307.

11. Lachman ME, Weaver SL. The sense of control as a moderator of social class differences in health and well-being. Journal of Personality and Social Psychology. 1998;74:763-773.

12. Berscheid E, Walster E, Bohrnstedt G. Body image. Psychology Today 1973;7:119-131.

13. McDonald PJ, Eilenfield VC. Physical attractiveness and the approach/ avoidance of self-awareness. Personality and Social Psychology Bulletin. 1980;6:391-395.

14. Albino JE, Tedesco LA, Kiyak HA. Esthetic issues in behavioral dentistry. Annals of Behavioral Medicine. 1990;12:148-155.

15. Shaw WC, Richmond S, Kenealy PM, Kingdon A, Worthington H. A 20year cohort study of health gain from orthodontic treatment: psychological outcome. American Journal of Orthodontics and Dentofacial Orthopedics. 2007;132:146-157.

16. Kok YV, Mageson P, Harradine NWT, Sprod AJ. Comparing a quality of life measure and the aesthetic component of the index of orthodontic treatment need (IOTN) in assessing orthodontic treatment need and concern. Journal of Orthodontics. 2004;31:312-318. 